\title{
The chimeric Japanese encephalitis/Dengue 2 virus protects mice from challenge by both dengue virus and JEV virulent virus
}

\section{Dear Editor}

Dengue fever (DF) is the most significant emerging arbovirus disease, which is caused by four distinct serotypes of Dengue viruses (DENV-1 to DENV-4). Serotype-specific immunity does not confer cross-protection against secondary infections from heterologous serotypes, which leads to dengue haemorrhagic fever (DHF) and dengue shock syndrome (DSS) as a result of antibody-dependent enhancement (ADE) of infection that is mediated by non-neutralizing, cross-reactive antibodies (Kliks et al., 1989). The intense efforts to develop a safe and efficient DENV vaccine have involved using a variety of traditional biological technologies in the past 50 years (Durbin et al., 2005; McArthur et al., 2008), yet no commercial DENV vaccine has been generated until now.

Developing DENV vaccine has been a high priority of the World Health Organization (WHO) (Chambers et al., 1997). Both Dengue virus and Japanese encephalitis virus (JEV) belong to the Flaviviridae. The JE attenuated live vaccine (SA14-14-2) was licensed in 1989 in China. Its safety and effectiveness have been demonstrated in large scale clinical surveillance and practical application of 600 million doses for more than 20 years in China and in Southeast Asian countries including Korea, Thailand and India (Yu, 2010). No vaccine associated encephalitis cases were reported for this JE vaccine, compared with 21 encephalitis cases associated with vaccination with the yellow fever (YF) vaccine 17D which is regarded as a safety vaccine (Monath et al., 1999). In particular, its unique profile of attenuated neurovirulence stability ensures the safety of the JE live vaccine and enables the utility of this live vaccine strain as a vector to develop vaccines against other Flaviviruses. This strategy has become feasible with the advent of reverse genetic method that has been used to construct chimeric flaviviruses (Pletnev et al., 2002; Mathenge et al., 2004). This approach has been used to prepare chimeric viruses YF/DENV (1-4) and such viruses exhibit high immunogenicity and low virulence (Chambers et al., 2003; Guirakhoo et al., 2004).

In this study, the prM/E genes of JEV SA14-14-2 in the plasmid pACNR-JEV were replaced with the prM/E gene from the DENV-2 PUO-218 strain to generate the plasmid
pACNR-JE/DENV-2 with the method reported by $\mathrm{Li}$ and Yang (Li et al., 2014; Yang et al., 2016). Results show that the restriction enzyme digestion of pACNR-JE/DENV-2 yielded the DNA fragments that were resolved by electrophoresis as expected. The chimeric virus JE/DENV-2 was produced by transfecting viral RNA into the BHK21 cells. The titre of the chimeric virus was approximately 3.3 $\log _{10} \mathrm{PFU} / \mathrm{mL}$. The size of the plaques ranged from 1 to $2 \mathrm{~mm}$ in diameter, which was smaller than that of JEV SA14-14-2 (2 to $3 \mathrm{~mm}$ in diameter) but larger than that of the DENV-2 (0.5-1 mm in diameter) (Fig. 1A). The results indicate that the chimeric virus is more like dengue virus rather than JEV and it is possible that the prM/E determines the size and formation of the plaque (Li et al., 2013).

As a vaccine candidate, it must be stable in passaging process. Sequence of the produced chimeric viruses from passage 1th to passage 14th was determined, which all contained the 10,959 nucleotides in the complete genome of the engineered JE/DENV-2 virus. No mutation was detected prior to passage 10th. 4 mutations were detected from passage 11 th to 14 th. They are at nucleotide position 39 (A to $\mathrm{T}$ ) in the $5^{\prime}$ UTR, position 1,531 (C to $\mathrm{T}$ ) in the structural protein $E$ region resulting in a serine to phenylalanine mutation, position 3,511 (A to $\mathrm{T}$ ) in the non-structural protein region resulting in a histidine to leucine mutation, and position 3,468 (A to $C$ ) in the non-structural protein region resulting in a methionine to leucine mutation.

Indirect immunofluorescence staining was adopted to detect the expression of viral proteins from chimeric virus JE/DENV-2 and the parental viruses JEV SA14-14-2 and DENV-2. The results showed that monoclonal antibody against the DENV-2 E protein detected the expression of the DENV-2 E protein in BHK21 cells that were infected with the DENV-2 virus and chimeric virus JE/DENV-2, but not with JEV (Fig. 1B top panel). Monoclonal antibody against the JEV $E$ protein detected the expression of JEV $E$ protein only in BHK21 cells infected by the JEV SA14-14-2 virus (Fig. 1B mid panel). Monoclonal antibody against the JEV NS1 protein detected the expression of JEV NS1 protein in cells infected by JEV SA14-14-2 or JE/DENV-2, but not by DENV-2 virus (Fig. 1B bottom panel). These results confirm the 


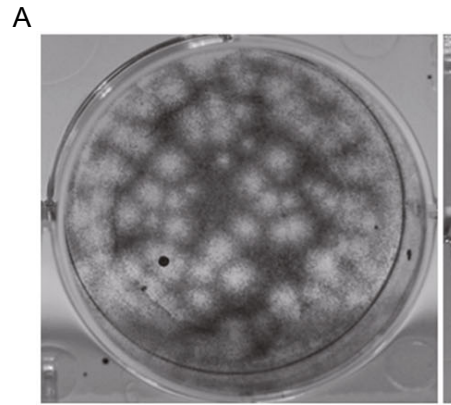

SA14-14-2

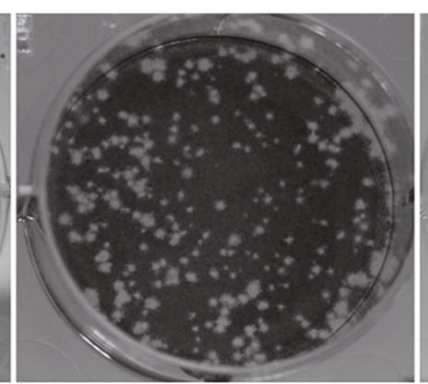

DENV-2

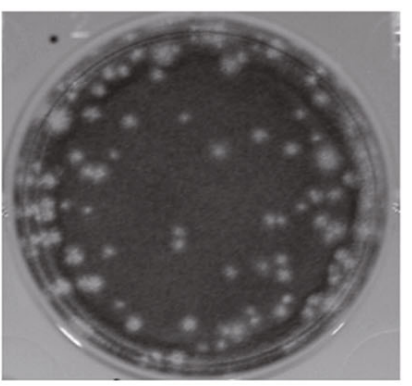

JE/DENV-2

B

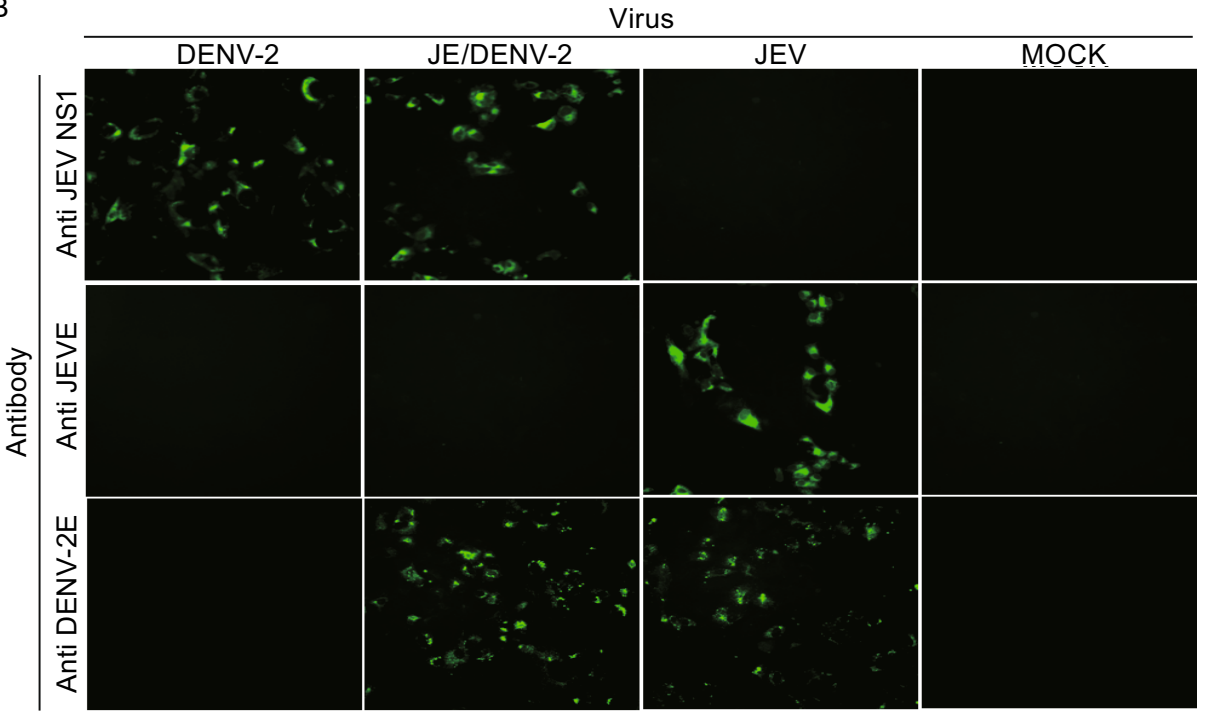

Figure 1. The diameter of plaque and viral proteins expression of JEV, JE/DENV-2 and DENV-2 in BHK21 cells. (A) The formation and diameter of plaques in BHK21 cells. (B) BHK21 cells were infected with each of these three viruses. Immunofluorescence staining was performed using antibodies recognizing DENV-2 E protein (1:10 dilution, top panel), JEV E protein (1:10 dilution, mid panel), or JEV NS1 protein (1:10 dilution, bottom panel). Mock represents no virus infection. DENV, dengue virus; JEV, Japanese encephalitis virus; JE, Japanese encephalitis; E, envelope protein; NS: non-structural protein.

successful expression of DENV-2 E protein from the chimeric virus JE/DENV-2 and indicate that the SA14-14-2 is a good vector to express exogenous antigen (Li et al., 2013; Li et al., 2014; Yang et al., 2016).

The growth kinetics of the JE/DENV-2 virus were determined by infecting PHK, Vero or C6/36 cells and the results were compared to the replication data of the DENV-2 virus NGC strain and the JEV SA14-14-2 virus. In PHK cells, the JE/DENV-2 propagated to lower titres at all time points compared to JEV which reached the peak titre of 7.3 $\log _{10} \mathrm{PFU} / \mathrm{mL}$ at $48 \mathrm{~h}$ post-infection. Instead, JE/DENV-2 exhibited a replication profile that was similar to that DENV-2. The peak titres of JE/DENV-2 and DENV-2 were 4.5 $\log _{10} \mathrm{PFU} / \mathrm{mL}$ and $5.4 \log _{10} \mathrm{PFU} / \mathrm{mL}$ at $36 \mathrm{~h}$, respectively (Fig. 2A). In Vero cells, JE/DENV-2 grew more rapidly than DENV-2, yet the titres of JE/DENV-2 were generally low with a peak value of $3.7 \log _{10} \mathrm{PFU} / \mathrm{mL}$ in $120 \mathrm{~h}$ despite of the different conditions that we tested. In contrast, JEV SA14-14-2 reached a peak titre of $6.6 \log _{10} P F U / m L$ at $96 \mathrm{~h}$ postinfection. DENV-2 replicated very slowly at the early time points compared to JEV, but was able to reach a peak titre of approximately $6.2 \log _{10} \mathrm{PFU} / \mathrm{mL}$ at $120 \mathrm{~h}$ (Fig. 2B). In C6/36 cells, JE/DENV-2 propagated less efficiently than JEV and DENV-2, and reached its peak titre of approximately 4.8 at $48 \mathrm{~h}$ post-infection compared to the peak titre of 7.2 $\log _{10} \mathrm{PFU} / \mathrm{mL}$ for JEV SA14-14-2 at $72 \mathrm{~h}$ and the peak titre of $6.0 \log _{10} \mathrm{PFU} / \mathrm{mL}$ for DENV-2 at $72 \mathrm{~h}$ (Fig. $2 \mathrm{C}$ ). We also investigated the growth kinetics of JE/DENV-2 with different M.O.I. in PHK cells. The virus reached its peak titre of 4.9 $\log _{10} \mathrm{PFU} / \mathrm{mL}$ at $24 \mathrm{~h}$ post-inoculation with the $0.025 \mathrm{M} . \mathrm{O} . \mathrm{I}$. and the peak titre of $5.5 \log _{10} \mathrm{PFU} / \mathrm{mL}$ at $48 \mathrm{~h}$ post-inoculation with the 0.005 M.O.I. and the 0.001 M.O.I. (Fig. 2D). In conclusion, the chimeric virus JE/DENV-2 propagates less efficiently compared to its parental virus JEV and exhibits a stronger or similar replication capacity compared to DENV-2.

Neurovirulence of the JE/DENV-2 virus was evaluated via the intra-cerebral (i.c.) route inoculation of 4-week old Kun-Min mice. The neurovirulence result showed that $\mathrm{LD}_{50}\left(\log _{10} \mathrm{PFU}\right)$ of JE/DENV-2 was 2.7. The JE live vaccine strain SA14-14-2 was also tested as the control, which did not show any 

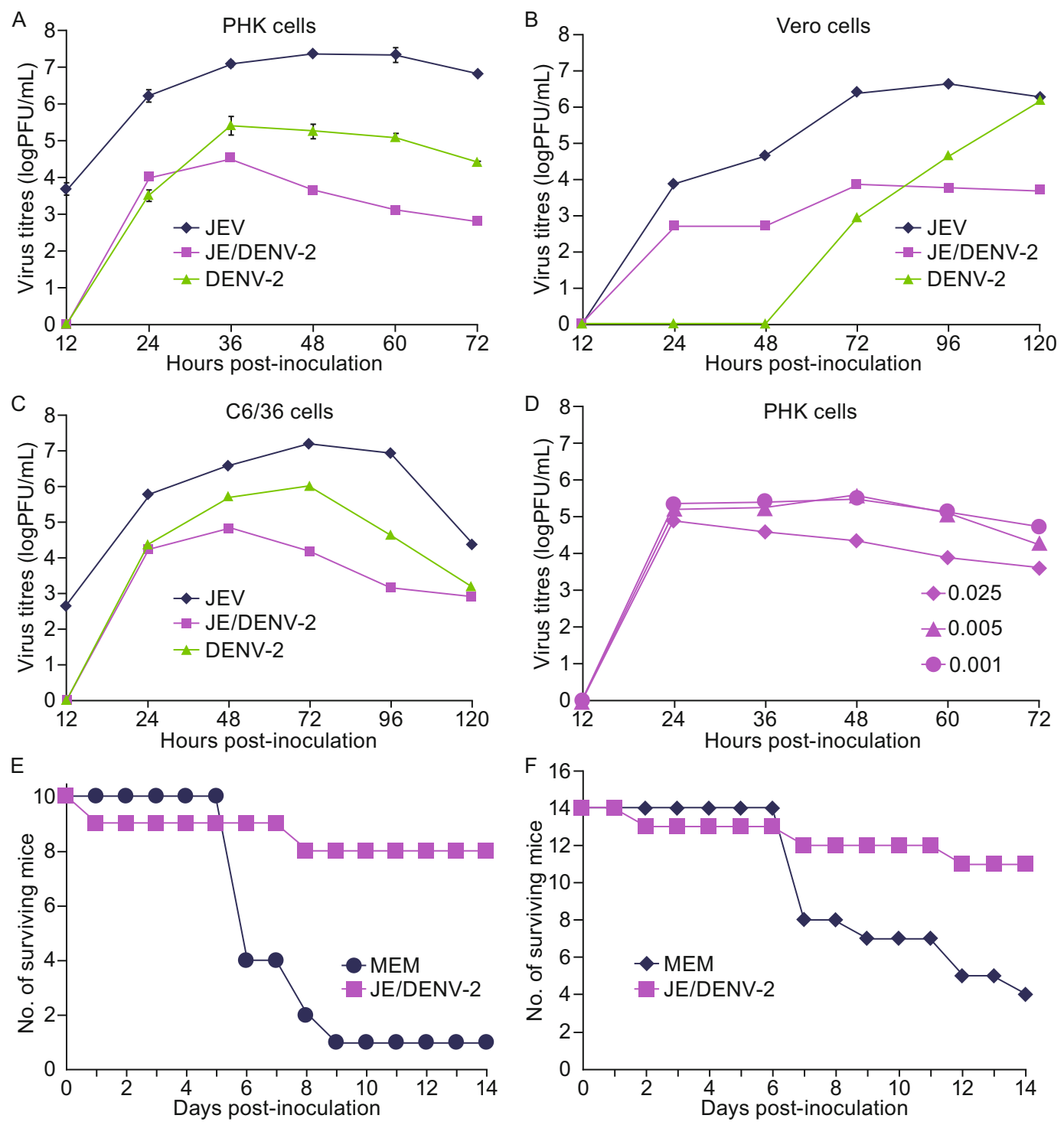

Figure 2. The growth curve of the chimeric virus in PHK, Vero and C6/36 cells and the chimeric virus JE/DENV-2 confers protection against neuroadapted DENV-2 virus (New Guinea strain, NGC) and JE virulent virus JEV/SA14. The growth curve of DENV-2, JE/DENV-2 and JEV in PHK cells (A), Vero cells (B), C6/36 cells (C) and PHK cells (D) (different M.O.I.). Mice were immunized with JE/DENV-2 via the i.p. route, or mock immunized (MEM). One month after immunization, mice were challenged via the i.c. route with neuroadapted DENV-2 NGC strain virus (shown in E) or via i.p. inoculation with JEV/SA14 virus (shown in F). The number of survived mice at each time point was recorded and plotted. M.O.I., multiplicity of infection; MEM, minimum essential medium; PHK, primary hamster kidney.

neurovirulence effect in mice. This result comes as a surprise, because both the parental viruses are avirulent to mice. No mouse died in the neuroinvasiveness test performed with JE/DENV-2 or SA14-14-2.

All mice used for immunogenicity study did not exhibit any sign of illness or mortality over the period of observation. The sera were treated for PRNT as described in other research. All mice immunized with the JE/DENV-2 chimeric virus were seroconverted to produce anti-DENV-2 antibody, based on the antibody titre value of 1:10. Antibody titre from each group was pooled to calculate geometric mean titres (GMTs). From week 1, 3, 5, 7, 9 and week 11 post boosting, the GMT reached $80,92,92,80,106$ and 184 , respectively. Differences of GMTs over this interval were not statistically significant.

We then investigated whether the JE/DENV-2 chimeric virus is able to induce protection against neuroadapted DENV-2 virus (NGC strain). For this purpose, mice were immunized with the JE/DENV-2 virus of $4.5 \log _{10} P F U$ via intra-peritoneal route (i.p.), followed by challenging via the i.c. route with the DENV-2 NGC strain of $4.5 \log _{10} P F U$ one month 
after immunization. The mice were observed for 2 additional weeks. None of these mice manifested any signs of illness for the one-month period before virus challenge. Results show that eight of nine immunized mice resisted the challenge of neuroadapted DENV-2 NGC strain, however, nine of ten mock-immunized mice succumbed to the challenge (Fig. 2E).

Because the chimeric JE/DENV-2 virus was constructed using JE SA14-14-2 as the vector, we suspected that mice immunized with JE/DENV-2 might also be protected from the challenge with JE virulent virus JEV/SA14, in which the prM/E gene of JEV SA14-14-2 was replaced with that of JEV wild type SA14. Some studies reported that antibody to JEV NS1 protein can provide immune protection against wild type JEV (Li et al., 2012). To test this possibility, mice were immunized with JE/DENV-2 and then challenged via the i.p. route with JEV/SA14 of $6.6 \log _{10}$ PFU one month after immunization. The mortality rate was $15 \%$ in the immunized group compared to $71 \%$ in mock-immunize group (Fig. $2 \mathrm{~F}$ ). The results are according with Li's results (Li et al., 2013). Together, these data demonstrate that immunization of mice with the chimeric virus JE/DENV-2 elicits strong protection against infection by either neuroadapted DENV-2 or virulent JEV/SA14.

In summary, results of this study demonstrate that the JE live vaccine virus serves as a promising vector for engineering flavivirus vaccines. The chimeric virus causes neurovirulence to mice via i.c. inoculation although the parent virus is avirulent and the reason need to be elucidated in the following work, meanwhile the neurovirulence in primate of chimeric virus need to be studied. Most importantly, we found that JE/DENV-2 may induce immunity protection against not only DENV-2 but also JEV. This will provide new sight to design vaccine to against Flavivirus.

\section{FOOTNOTES}

LYH and YHQ conceived and designed the studies. YJ, LZS, LH and $Z Y$ performed the experiments. ZXW contributed reagents, materials and analysis tools. YJ and LYH analyzed the data. YJ prepared the manuscript. We would like to thank Professor $\mathrm{Yu}$ Yong-xin for providing DENV-2 virus (NGC strain). This work was supported by the National High Technology Research and Development Program (863 Program) (No. 2012AA02A401). The funder had no role in study design, data collection and analysis, decision to publish, or preparation of the manuscript.

Jian Yang, Huiqiang Yang, Zhushi Li, Hua Lin, Yu Zhao, Wei Wang, Shuai Tan, Xianwu Zeng, and Yuhua Li declare that they have no conflict of interest.

The experimental protocols involving mice were approved by the Animal Experimental Committee of Chengdu Institute of Biological Products Co. Ltd. All institutional and national guidelines for the care and use of laboratory animals were followed.

Electronic supplementary material The online version of this article (doi:10.1007/s13238-016-0363-5) contains supplementary material, which is available to authorized users.
Jian Yang ${ }^{1,2}$, Huiqiang Yang ${ }^{1}$, Zhushi Li ${ }^{1}$, Hua Lin ${ }^{1}$, Yu Zhao ${ }^{1}$, Wei Wang ${ }^{1}$, Shuai Tan ${ }^{1}$, Xianwu Zeng ${ }^{1}$, Yuhua $\mathrm{Li}^{3,4 \bowtie}$

${ }^{1}$ Department of Viral Vaccine, Chengdu Institute of Biological Products Co., Ltd., Chengdu 610023, China

2 Department of Microbiology and Immunology, North Sichuan Medical College, Nanchong 637007, China

${ }^{3}$ Department of Arboviruses Vaccine, National Institute for Food and Drug Control, Beijing 100050, China

${ }^{4}$ State Key Laboratory of Biotherapy and Cancer Center, West China Hospital, Sichuan University and Collaborative Innovation Center for Biotherapy, Chengdu 610000, China

$\bowtie$ Correspondence: liyuhua@nifdc.org.cn (Yuhua Li)

\section{OPEN ACCESS}

This article is distributed under the terms of the Creative Commons Attribution 4.0 International License (http://creativecommons.org/ licenses/by/4.0/), which permits unrestricted use, distribution, and reproduction in any medium, provided you give appropriate credit to the original author(s) and the source, provide a link to the Creative Commons license, and indicate if changes were made.

\section{REFERENCES}

Chambers TJ, Tsai TF, Pervikov Y, Monath TP (1997) Vaccine development against dengue and Japanese encephalitis: report of a World Health Organization meeting. Vaccine 15:14941502

Chambers TJ, Liang Y, Droll DA, Schlesinger JJ, Davidson AD, Wright PJ, Jiang X (2003) Yellow fever virus/dengue-2 virus and yellow fever virus/dengue-4 virus chimeras: biological characterization, immunogenicity, and protection against dengue encephalitis in the mouse model. J Virol 77:3655-3668

Durbin AP, Whitehead SS, McArthur J, Perreault JR, Blaney JE Jr, Thumar B, Murphy BR, Karron RA (2005) rDEN4delta30, a live attenuated dengue virus type 4 vaccine candidate, is safe, immunogenic, and highly infectious in healthy adult volunteers. J Infect Dis 191:710-718

Guirakhoo F, Pugachev K, Zhang Z, Myers G, Levenbook I, Draper K, Lang J, Ocran S, Mitchell F, Parsons M et al (2004) Safety and efficacy of chimeric yellow Fever-dengue virus tetravalent vaccine formulations in nonhuman primates. J Virol 78:4761-4775

Kliks SC, Nisalak A, Brandt WE, Wahl L, Burke DS (1989) Antibodydependent enhancement of dengue virus growth in human monocytes as a risk factor for dengue hemorrhagic fever. Am J Trop Med Hyg 40:444-451

Li Y, Counor D, Lu P, Duong V, Yu Y, Deubel V (2012) Protective immunity to Japanese encephalitis virus associated with antiNS1 antibodies in a mouse model. Virol J 9:135

Li XF, Deng YQ, Yang HQ, Zhao H, Jiang T, Yu XD, Li SH, Ye Q, Zhu SY, Wang HJ et al (2013) A chimeric dengue virus vaccine using Japanese encephalitis virus vaccine strain SA14-14-2 as backbone is immunogenic and protective against either parental virus in mice and nonhuman primates. J Virol 87:13694-13705 
Li Z, Yang $\mathrm{H}$, Yang J, Lin H, Wang W, Liu L, Zhao Y, Liu L, Zeng X, Yu $Y$ et al (2014) Construction and preliminary investigation of a novel dengue serotype 4 chimeric virus using Japanese encephalitis vaccine strain SA14-14-2 as the backbone. Virus Res 191:10-20

Mathenge EG, del Parquet C, Funakoshi MY, Houhara S, Wong PF, Ichinose A, Hasebe F, Inoue S, Morita K (2004) Fusion PCR generated Japanese encephalitis virus/dengue 4 virus chimera exhibits lack of neuroinvasiveness, attenuated neurovirulence, and a dual-flavi immune response in mice. J Gen Virol 85:25032513

McArthur JH, Durbin AP, Marron JA, Wanionek KA, Thumar B, Pierro DJ, Schmidt AC, Blaney JE Jr, Murphy BR, Whitehead SS (2008) Phase I clinical evaluation of rDEN4Delta30-200,201: a live attenuated dengue 4 vaccine candidate designed for decreased hepatotoxicity. Am J Trop Med Hyg 79:678-684

Monath TP, Soike K, Levenbook I, Zhang ZX, Arroyo J, Delagrave S, Myers G, Barrett AD, Shope RE, Ratterree M et al (1999)
Recombinant, chimaeric live, attenuated vaccine (ChimeriVax) incorporating the envelope genes of Japanese encephalitis (SA14-14-2) virus and the capsid and nonstructural genes of yellow fever (17D) virus is safe, immunogenic and protective in non-human primates. Vaccine 17:1869-1882

Pletnev AG, Putnak R, Speicher J, Wagar EJ, Vaughn DW (2002) West Nile virus/dengue type 4 virus chimeras that are reduced in neurovirulence and peripheral virulence without loss of immunogenicity or protective efficacy. Proc Natl Acad Sci USA 99:30363041

Yang H, Li Z, Lin H, Wang W, Yang J, Liu L, Zeng X, Wu Y, Yu Y, Li Y (2016) A novel dengue virus serotype 1 vaccine candidate based on Japanese encephalitis virus vaccine strain SA14-14-2 as the backbone. Arch Virol 161:1517-1526

Yu Y (2010) Phenotypic and genotypic characteristics of Japanese encephalitis attenuated live vaccine virus SA14-14-2 and their stabilities. Vaccine 28:3635-3641 